\title{
Effect of awareness programs and travel-blocking operations in the control of HIV/AIDS outbreaks: a multi-domains SIR model
}

\author{
Omar Zakary*, Abdelilah Larrache, Mostafa Rachik and Ilias Elmouki
}

\author{
"Correspondence: \\ zakaryma@gmail.com \\ Department of Mathematics and \\ Computer Science, Hassan II \\ University Of Casablanca, BP 7955, \\ Sidi Othman, Casablanca, Morocco
}

\begin{abstract}
The main goal of this article is to exhibit the importance of awareness programs and travel-blocking operations, in the prevention of HIV/AIDS outbreaks, based on a multi-domains SIR epidemic model. The model devised describes the spatial-temporal spread of HIV/AIDS, in p neighboring domains, taking into account the epidemiological diversity of their populations. For a first case of the study, we focus on discussing the benefits of awareness campaigns that aim to raise public consciousness among susceptible people, about the danger of HIV/AIDS. Thus, for the reason that intra-domains interventions are not always sufficient to prevent people belonging to a domain (neighborhood, town or city for example), from a rapid expansion of HIV/AIDS, we propose the travel-blocking strategy, as a second approach presenting inter-domains interventions that health-policy makers could follow, by restricting the number of suspicious people that can participate in spreading HIV/AIDS via travel, coming from domains at high-risk of infection to enter domains with lower risk. The optimal control theory, based on Pontryagin's maximum principle, is applied twice in this paper, for the characterizations of the awareness and travel-blocking controls. The numerical results associated to the multi-points boundary value problems are obtained based on the Forward-Backward Sweep Method combined with progressive-regressive Runge-Kutta fourth-order schemes.
\end{abstract}

Keywords: HIV/AIDS; SIR model; multi-domains model; optimal control; awareness programs; travel-blocking

\section{Introduction}

Human immunodeficiency virus infection and acquired immune deficiency syndrome (HIV/AIDS) is among epidemics that affect people either living or working in extremely poor regions of the entire terrestrial globe $(25,800,000$ people in sub-Saharan African countries alone are living with HIV), while some important HIV/AIDS infection rates can be observed also in the most affluent communities [1, 2]. Ayiro et al., in [3], aimed to explain the mutual relationship which exists between education and HIV/AIDS and how they influence each other. Here we focus on the impact of education in HIV/AIDS based on the organization of many educational workshops and by following different awareness programs. Sometimes, in the case of the most-deadly diseases such as HIV/AIDS, it is

(c) 2016 Zakary et al. This article is distributed under the terms of the Creative Commons Attribution 4.0 International License (http://creativecommons.org/licenses/by/4.0/), which permits unrestricted use, distribution, and reproduction in any medium, provided you give appropriate credit to the original author(s) and the source, provide a link to the Creative Commons license, and indicate if changes were made. 
in fact believed that awareness may represent the most effective social vaccine for controlling this epidemic, in that it could help to increase the proportion of consciousness amongst children and adults [4]. An awareness campaign can play a significant role to influence young people for helping them to change their attitudes and sexual behaviors, and for expanding their knowledge about HIV/AIDS infection and prevention. Educational workshops may also represent an occasion to explain to students the importance of having regular blood sampling, and to present them treatment and care that are already available if necessary. In short, educational workshops and awareness programs could represent powerful plans and strategic actions in the war against HIV/AIDS in many different countries [5-10]. José Catàlan et al. highlighted [11], the benefits of awareness programs that can be advertised by the media, and led by some health associations and governmental institutions, to intervene as major players to promote the civil society in taking more actions and making many efforts to decrease the HIV infection rate regionally and then worldwide. The evolution of infectious diseases has been described and analyzed by many mathematical modelers, while conventional mathematical modeling of the spread of the disease has usually ignored or underestimated the importance of the spatial exposure of an epidemic (see [12-16] and references therein). The importance of modeling the spatial spread of an infectious disease can be highlighted by considering the Ebola outbreaks in several sub-Saharan African countries [17]. Guinean infected people moved from one city to another, and sometimes traveled to neighboring countries. After some infected cases were reported in March 2014 in Liberia linked to some foreigners who entered the country, it took some time until the political decision was made of banning all movements in Guinea in order to help contain the spread of the virus and prevent it from reaching Liberia and other regions [18]. During that period, the infected cases had already been divided among a large number of countries. The problem was exacerbated. Then, for controlling the spatial spread of the disease, it is necessary to consider all parts that people can visit. Thus, it is essential to integrate the spatial dynamics in models that attempt to describe the spread of infectious diseases.

Optimal control theory has been applied to many diseases, mainly tuberculosis [1923], malaria [24, 25], HIV [26-33], hepatitis [34, 35], vector borne diseases [36], cancer [37-42], and other diseases [43-49]. The vaccination and treatment interventions in the aforementioned studies, related to infectious diseases, were optimized for the case of a particular domain (one city for instance), rather than talking about an optimal control strategy in the case of more than one domain especially when a disease is transmitted by people who travel from one domain to another. Hence, this work has a novel control application in addition to providing a framework to analyze spatial control strategies.

The aim of this work is to consider an HIV/AIDS multi-domains SIR epidemic model and to set up an optimal control approach for it. In order to show the effectiveness of awareness strategies on reducing the number of HIV/AIDS infectives, a control function is introduced into the model to represent the effectiveness rate of awareness programs on susceptible population. Hence, the goal of the first optimal control (awareness program) strategy is to minimize the infected and susceptible individuals, and to maximize the total number of recovered individuals in a specific domain (neighborhood, town or city) by using the minimum possible cost of applying this control and simultaneously, to investigate the sensitivity of the susceptible individuals by this control. We illustrate the numerical results, obtained from the applied optimal control theory (i.e. Pontryagin's maximum 
principle), and we show the effect of the percentage of the awareness variable (control) on minimizing the number of susceptible and infected individuals and increasing the number of the removed ones. Second, in order to show the importance of considering the spatial spread of the epidemic, we show in the same section of simulations the influence of values of adequate contact proportions of domains at high-risk on a neighboring domain that is characterized by less risk of infection. A second control variable that characterizes the travel-blocking operation is added, and hereby it is attempted to block contacts between susceptibles of the controlled domain (by awareness) and infected individuals of domains at high-risk of infection with HIV/AIDS. After deriving the optimality system associated to the multi-domains SIR model, we find two optimal control strategies. The numerical results are simulated using the Forward-Backward Sweep Method with integrated progressive-regressive Runge-Kutta fourth-order schemes associated to every single domain.

In the following sections, we begin by describing the different components of the suggested SIR epidemic model for HIV/AIDS, and afterward we choose to minimize two objective functionals. The sought solutions (optimal controls) related to the chosen minimization criteria are simulated with their associated states in the numerical results sections. For the case when only the awareness control is used, the optimal control approach is presented in Section 2. As regards the travel-blocking approach, the analysis of the optimal control problem and numerical simulations are given in Section 3. Finally, we conclude the paper in Section 4.

\section{Presentation of the model with awareness control only}

First of all, we should note that we suggested in [26] an SIR mathematical model with an optimal control strategy that was aiming to show the impact of awareness programs in the prevention of HIV/AIDS in only one domain (general case study that can be applied to only one country, city, etc.). Here, we extend the study done in [26] by considering $p$ domains (neighborhoods, towns or cities) of the spread of the HIV/AIDS epidemic. The $p$ domains are characterized by different parameters of infection in order to show the influence of domains at high risk of infection with HIV/AIDS, on a domain that is characterized by a lower infection rate.

Consider an SIR model for HIV transmission in a population of individuals, and we assume that there are $p$ geographical sub-domains of the studied domain $\Omega$. Let $\Omega=\bigcup_{j=1}^{p} \Omega_{j}$. According to the disease transmission mechanism, the host population of each domain $\Omega_{j}$ is grouped into three epidemiological compartments, $S^{\Omega_{j}}$ susceptible individuals, $I^{\Omega_{j}}$ infected individuals and $R^{\Omega_{j}}$ individuals removed from the disease, i.e. people who are sexually inactive, or persons who have taken the necessary precautions to avoid infection. We assume that the susceptible individuals are not yet infected with HIV but can be infected through sexual contacts with infectives or become aware of the danger of the disease and transfer to the removed class. In addition to the death and recruitment, there are population movements among those three epidemiological compartments. We assume that the recruited individuals (by birth and immigration), in $\Omega_{j}$, are constant and enter the susceptible compartment. In addition, we suppose that individuals who are out of their domain do not give birth, and so birth occurs in the home domain at a per capita rate $\Gamma_{j}>0$. Further, it is assumed that death takes place anywhere and the number of people removed from each class due to natural causes such as death (not HIV or AIDS related) 
is proportional to the number of individuals in the compartment, $\mu_{j} S^{\Omega_{j}}, \mu_{j} I^{\Omega_{j}}$ and $\mu_{j} R^{\Omega_{j}}$, where $\mu_{j}>0$ will be called the natural death rate for historical reasons, which is constant. Individuals in the infective compartment can remain infectives, or be transferred to the removed compartment (they are no longer sexually infectives) at a rate $\gamma_{j}{ }^{\Omega_{j}}$, where $\gamma_{j}>0$ is the removal rate which is a constant, or die. In fact, individuals in the removed compartment never leave the compartment unless they die.

Infection transmission is assumed to occur between individuals present in a given domain $\Omega_{j}$, and it is given by

$$
\sum_{k=1}^{p} \beta_{j k} \frac{I^{\Omega_{k}}}{I^{\Omega_{k}}+S^{\Omega_{k}}} S^{\Omega_{j}},
$$

where $\frac{I^{\Omega_{k}}}{I^{\Omega_{k}}+S^{\Omega_{k}}}$ is the proportion of infected individuals to sexually active individuals in $\Omega_{k}$, and $\beta_{j k}>0$ is the proportion of adequate contacts in domain $\Omega_{j}$ between a susceptible from $\Omega_{j}$ and an infective from another domain $\Omega_{k}$, which is a constant.

One of the prime objectives of this work is to study the spatial spread of HIV/AIDS in several domains. Thus, in the case of three domains $\Omega_{j}, \Omega_{l}$, and $\Omega_{k}$, and where we need to control $\Omega_{j}$, if we suppose for instance $\beta_{j k}>0$ and $\beta_{j l}=0$, i.e. $\Omega_{j}$ is not accessible from $\Omega_{l}$, there would be no reason to consider $\Omega_{l}$, which is a case usually avoided in mathematical modeling because if one parameter is zero, there is no need to add it since the first time. Since all $\Omega_{j}$ are supposed to be interconnected by any kind of movements, it is assumed that $\beta_{j k}>0$ for all $j$ and $k$.

The simplest example we can give here is the case of two infected individuals $X$ and $Y$ from a domain $\Omega_{k}$ and who would travel separately toward the two domains $\Omega_{j}$ and $\Omega_{l}$. It is then obvious that $X$ and $Y$ can infect susceptibles from $\Omega_{j}$ and $\Omega_{l}$ with different proportions $\beta_{j k}$ and $\beta_{l k}$ at the same time.

For the sake of readability, hereafter, we will use the following notations:

$$
\begin{aligned}
S^{\Omega_{j}} & \rightarrow S^{j}, \\
I^{\Omega_{j}} & \rightarrow I^{j}, \\
R^{\Omega_{j}} & \rightarrow R^{j}, \\
\theta^{\Omega_{j}} & \rightarrow \theta^{j} \quad \text { (explained further below). }
\end{aligned}
$$

The following system of ODEs describes this multi-domains SIR model:

$$
\begin{aligned}
& \frac{d S^{j}}{d t}=\Gamma_{j}-\sum_{k=1}^{p} \beta_{j k} \frac{I^{k}}{I^{k}+S^{k}} S^{j}-\mu_{j} S^{j}-\theta^{j} S^{j}, \\
& \frac{d I^{j}}{d t}=\sum_{k=1}^{p} \beta_{j k} \frac{I^{k}}{I^{k}+S^{k}} S^{j}-\gamma_{j} I^{j}-\mu_{j} I^{j}, \\
& \frac{d R^{j}}{d t}=\gamma_{j} I^{j}-\mu_{j} R^{j}+\theta^{j} S^{j},
\end{aligned}
$$

where $\theta^{j}$ represents the effectiveness of the awareness program in $\Omega_{j}$; it tries to increase the number of removed people and decreases the susceptible ones. 


\subsection{Optimal control problem}

We are interested in controlling the population of domain $\Omega_{j}$. Then the problem is to minimize the objective functional given by

$$
J\left(\theta^{j}\right)=\int_{0}^{t_{f}}\left(A_{1} I^{j}(t)-A_{2} R^{j}(t)+\frac{K}{2}\left(\theta^{j}(t)\right)^{2}\right) d t .
$$

The first terms represent the crucial goal of the awareness program, that is of reducing the number of the infected people, and increasing the removed ones, in the domain $\Omega_{j}$. The other term is the systemic cost of the awareness program. The positive constants $A_{1}$, $A_{2}$, and $K$ balance the values of the terms. Also the reason behind considering a finite time horizon is that such a control program is usually restricted to a limited time window.

We seek an optimal control $\theta^{j *}$ such that

$$
J\left(\theta^{j *}\right)=\min \left\{J\left(\theta^{j}\right) \mid \theta^{j} \in \Theta\right\}
$$

where

$$
\Theta=\left\{\theta^{j} \text { measurable, } \theta^{\min } \leq \theta^{j}(t) \leq \theta^{\max }, t \in\left[0, t_{f}\right]\right\},
$$

where $\left.\left(\theta^{\min }, \theta^{\max }\right) \in\right] 0,1\left[{ }^{2}\right.$. The necessary conditions that an optimal control problem must satisfy come from Pontryagin's maximum principle [50]. This principle converts (4)(1)-(3) into a problem of minimizing point-wise a Hamiltonian $H$, with respect to $\theta^{j}$,

$$
\begin{aligned}
H= & A_{1} I^{j}(t)-A_{2} R^{j}(t)+\frac{K}{2}\left(\theta^{j}(t)\right)^{2} \\
& +\lambda_{1}^{j}\left[\Gamma_{j}-\sum_{k=1}^{p} \beta_{j k} \frac{I^{k}}{I^{k}+S^{k}} S^{j}-\mu_{j} S^{j}-\theta^{j} S^{j}\right] \\
& +\lambda_{2}^{j}\left[\sum_{k=1}^{p} \beta_{j k} \frac{I^{k}}{I^{k}+S^{k}} S^{j}-\gamma_{j} I^{j}-\mu_{j} I^{j}\right] \\
& +\lambda_{3}^{j}\left[\gamma_{j} I^{j}-\mu_{j} R^{j}+\theta^{j} S^{j}\right] .
\end{aligned}
$$

Here $\lambda_{i}^{j}, i=1, \ldots, 3$, represent the adjoint functions of the state variables $S^{j}, I^{j}$, and $R^{j}$, to be determined suitably. We can verify the existence of the optimal control $\theta^{j *}$ based on the properties announced in the following theorem.

Theorem 1 There exist an optimal control $\theta^{j *}$ and corresponding solution, $S^{j *}, I^{j *}$, and $R^{j *}$ that minimize $J\left(\theta^{j}\right)$ over $\Theta$.

Proof Based on an existence result from Theorem III.4.1 from [51], the following conditions must be checked:

1. The set of control and corresponding state variables is non-empty.

2. The control $\Theta$ set is convex and closed.

3. The right hand side of the state system is bounded by a linear function in the state and control variables.

4. The integrand of the objective functional is convex on $\Theta$. 
5. There exist constant $c_{1}, c_{2}>0$, and $\beta>1$ such that the integrand $L\left(I^{j}, R^{j}, \theta^{j}\right)$ of the objective functional satisfies

$$
L\left(I^{j}, R^{j}, \theta^{j}\right) \geq c_{1}\left(\left|\theta^{j}\right|^{2}\right)^{\beta / 2}-c_{2} .
$$

In order to verify these properties, we use a result provided by Lukes [[52], Theorem 9.2.1, p.182] to give the existence of solutions of ODEs (1)-(3) with bounded coefficients, which gives condition 1 , and we note the solutions are bounded.

The $\Theta$ set satisfies condition 2 by definition.

Since our state system is bilinear in $\theta^{j}$, the right hand-side of (1)-(3) satisfies condition 3, using the boundedness of the solutions.

Finally, we can easily see that the integrand of our objective functional is convex and that there exist a constant $\beta>1$ and positive numbers $c_{1}$ and $c_{2}$ satisfying

$$
L\left(I^{j}, R^{j}, \theta^{j}\right) \geq c_{1}\left(\left|\theta^{j}\right|^{2}\right)^{\beta / 2}-c_{2} .
$$

By applying Pontryagin's maximum principle [50] and assuming the existence result for the optimal control from the previous Theorem 1, we obtain the following theorem.

Theorem 2 Given an optimal control $\theta^{j *}$ and corresponding solutions, $S^{j *}, I^{j *}$, and $R^{j *}$, that minimize $J\left(\theta^{j}\right)$ over $\Theta$, there exist adjoint functions, $\lambda_{1}^{j}(t), \lambda_{2}^{j}(t)$, and $\lambda_{3}^{j}(t)$, such that

$$
\begin{aligned}
& \dot{\lambda}_{1}^{j}=-\left[\left(\frac{\beta_{j j} S^{j *} I^{j *}}{\left(I^{*}+S^{j *}\right)^{2}}-\sum_{k=1}^{p} \beta_{j k} \frac{I^{k *}}{I^{k *}+S^{k *}}\right)\left(\lambda_{1}^{j}-\lambda_{2}^{j}\right)-\mu_{j} \lambda_{1}^{j}+\theta^{j}\left(\lambda_{3}^{j}-\lambda_{1}^{j}\right)\right], \\
& \dot{\lambda}_{2}^{j}=-\left[A_{1}+\frac{\beta_{j j} j^{j * 2}}{\left(I^{j *}+S^{j *}\right)^{2}}\left(\lambda_{2}^{j}-\lambda_{1}^{j}\right)-\left(\gamma_{j}+\mu_{j}\right) \lambda_{2}^{j}+\gamma_{j} \lambda_{3}^{j}\right], \\
& \dot{\lambda}_{3}^{j}=-\left[-A_{2}-\mu_{j} \lambda_{3}^{j}\right],
\end{aligned}
$$

with transversality conditions

$$
\lambda_{i}^{j}\left(t_{f}\right)=0, \quad i=1,2,3 .
$$

The following characterization holds:

$$
\theta^{j *}(t)=\max \left\{\min \left\{\left(\lambda_{1}^{j}(t)-\lambda_{3}^{j}(t)\right) \frac{S^{j *}(t)}{K}, \theta^{\max }\right\}, \theta^{\min }\right\} .
$$

Proof Applying Pontryagin's maximum principle [50], we obtain

$$
\begin{aligned}
\dot{\lambda}_{1}^{j} & =-\frac{d H}{d S^{j}} \\
& =-\left[\left(\frac{\beta_{j j} j^{*} I^{j *}}{\left(I^{*}+S^{j *}\right)^{2}}-\sum_{k=1}^{p} \beta_{j k} \frac{I^{k *}}{I^{k *}+S^{k *}}\right)\left(\lambda_{1}^{j}-\lambda_{2}^{j}\right)-\mu_{j} \lambda_{1}^{j}+\theta^{j}\left(\lambda_{3}^{j}-\lambda_{1}^{j}\right)\right], \quad \lambda_{1}^{j}\left(t_{f}\right)=0, \\
\dot{\lambda}_{2}^{j} & =-\frac{d H}{d I^{j}}=-\left[A_{1}+\frac{\beta_{j j} j^{j * 2}}{\left(I^{j *}+S^{j *}\right)^{2}}\left(\lambda_{2}^{j}-\lambda_{1}^{j}\right)-\left(\gamma_{j}+\mu_{j}\right) \lambda_{2}^{j}+\gamma_{j} \lambda_{3}^{j}\right], \quad \lambda_{2}^{j}\left(t_{f}\right)=0,
\end{aligned}
$$




$$
\dot{\lambda}_{3}^{j}=-\frac{d H}{d R^{j}}=-\left[-A_{2}-\mu_{j} \lambda_{3}^{j}\right], \quad \lambda_{3}^{j}\left(t_{f}\right)=0,
$$

evaluated at the optimal control $\theta^{j *}$ and the corresponding states $S^{j *}, I^{j *}$, and $R^{j *}$, which results in the stated adjoint system (5)-(7) and (8), [53]. By considering the optimality conditions,

$$
\frac{d H}{d \theta^{j}}=0,
$$

and solving for $\theta^{j}$, we find

$$
\frac{d H}{d \theta^{j}}=K \theta^{j}-\lambda_{1}^{j} S^{j}+\lambda_{3}^{j} S^{j}=0
$$

Taking into account the bounds on $\theta^{j *}$ in $\Theta$, we deduce that

$$
\theta^{j *}(t)=\max \left\{\min \left\{\left(\lambda_{1}^{j}(t)-\lambda_{3}^{j}(t)\right) \frac{S^{j *}(t)}{K}, \theta^{\max }\right\}, \theta^{\min }\right\} .
$$

\subsection{Numerical results}

We now present numerical simulations associated with our optimal system derived from the previous mathematical model. We write a code in MATLAB ${ }^{T M}$ and we simulate our results using different data. We solve the optimality systems using an integrated progressiveregressive Runge-Kutta fourth-order iterative scheme associated to every single domain. Such numerical procedures are called forward-backward sweep methods, where the state system with an initial guess is solved forward in time and then the adjoint system is solved backward in time. First, starting with an initial guess for the adjoint variables $\lambda_{1}^{j}, \lambda_{2}^{j}$ and $\lambda_{3}^{j}$, we solve the state equations by forward Runge-Kutta fourth-order schemes in time associated to every single domain $\Omega_{j}$. Then those state values are used to solve the adjoint equations by backward Runge-Kutta fourth-order schemes because of the transversality conditions $[19,54]$. Afterward, we updated the optimal control values using the values of state and costate variables obtained in the previous steps. Finally, we execute the previous steps till a tolerance criterion is reached.

In order to show the importance of our work, we choose the example when $p=2$, i.e., we consider two domains $\Omega_{1}$ and $\Omega_{2}$ with different parameters cited in Table 1. We try to control $\Omega_{1}$ by the control $\theta^{1 *}$ given by (9), which represents the effectiveness of the awareness program in this domain.

In Figure 1, we can observe that in the absence of any awareness control and in the presence of an epidemic that spreads in two domains characterized by different parameters, the number of infected individuals rises from $I_{0}^{1}=200$ and $I_{0}^{2}=100$ as initial conditions

Table 1 Parameters values of $\Gamma, \beta, \mu$, and $\boldsymbol{\gamma}$ utilized for the resolution of the SIR multi-domains differential system (1)-(3), and then leading to simulations obtained from Figure 1 to Figure 4 , with the initial conditions $S_{0}, I_{0}$, and $R_{0}$ associated to the two regions $\Omega_{1}$ and $\boldsymbol{\Omega}_{2}$

\begin{tabular}{rrrlllll}
\hline & $\boldsymbol{S ( 0 )}$ & $\boldsymbol{I ( 0 )}$ & $\boldsymbol{R ( 0 )}$ & $\boldsymbol{\Gamma}$ & $\boldsymbol{\beta}$ & $\boldsymbol{\mu}$ & $\boldsymbol{\gamma}$ \\
\hline$\Omega_{1}$ & 10,000 & 200 & 0 & 100 & 0.04 & 0.012 & 0.002 \\
$\boldsymbol{\Omega}_{2}$ & 9,000 & 100 & 0 & 180 & 0.05 & 0.013 & 0.001 \\
\hline
\end{tabular}


(a)

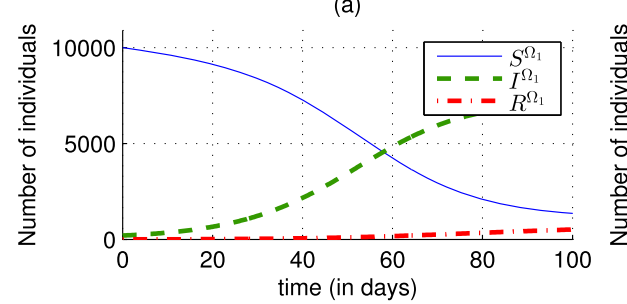

(b)

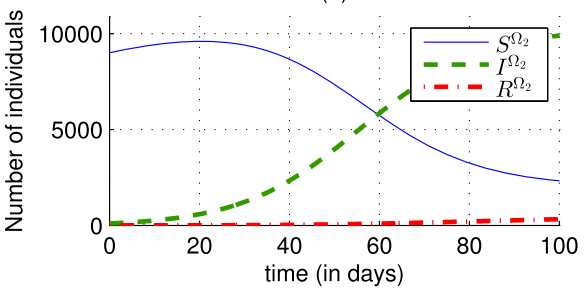

Figure 1 States of system (1)-(3) without controls. (a) Domain $\Omega_{1}$. (b) Domain $\Omega_{2}$.

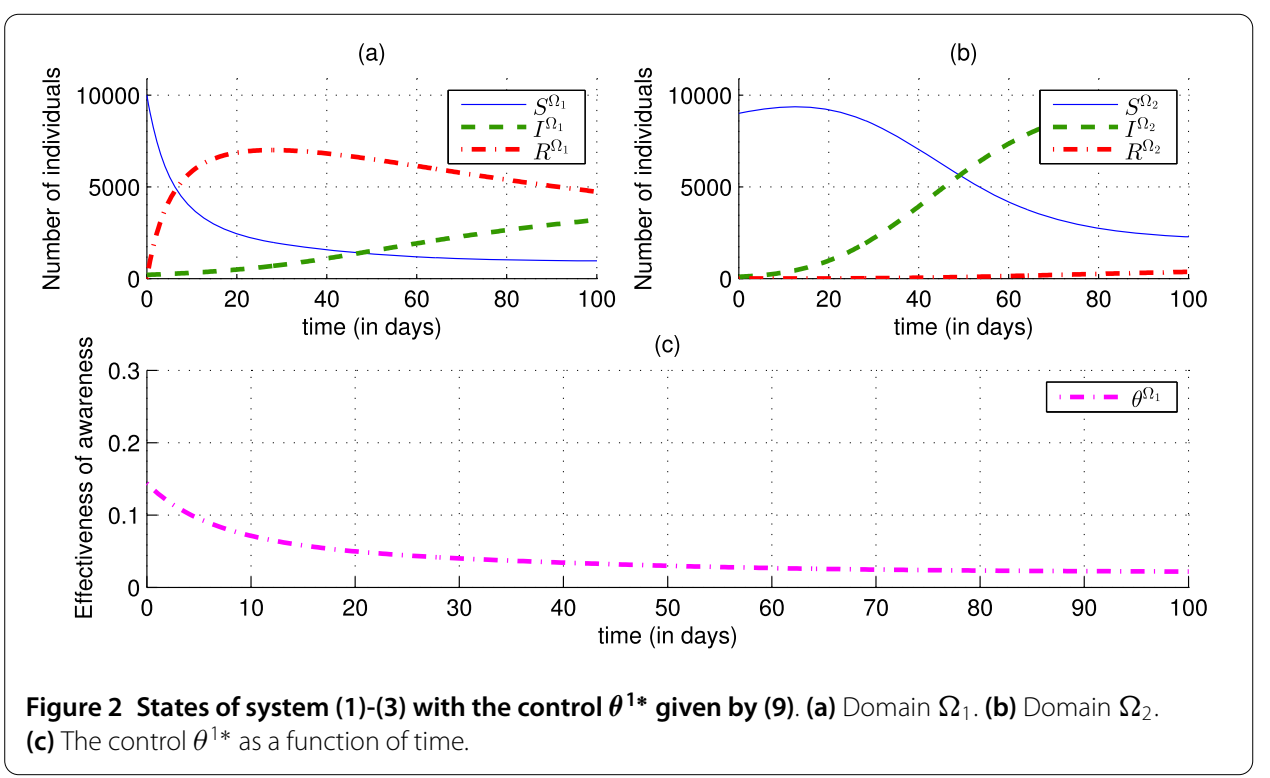

to 6,200 and 10,000 in the two domains, respectively. Once an awareness control is introduced in the system (1)-(3), particularly in the equations that describe the dynamics of $S^{1}$, $I^{1}$, and $R^{1}$ functions associated to the first domain, we can deduce its effect on decreasing the number of infected people in Figure 2, from 6,190 when there was yet no awareness control strategy, to 3,800 when there is the control $\theta^{1}$. One of the major benefits of that control is to increase the number of the removed people, and this can be observed in case (a) of Figure 2, where the number of the removed people becomes approximately equal to 6,500, and then begins to decrease to a value close to 5,000, which shows the proportionality between the effectiveness of the awareness program and the number of removed individuals. That can obviously prove the effectiveness of this awareness strategy in the first domain with a rate that varies in Figure 2(c), from a value equal to 0.14 toward a value equal to 0.01 , and this also proves that by a control taking only a nonzero value close to 0 , we can reach our goal with a significant number of the removed people.

We observe in Figure 3, as the control severity weight $K$ is small, the control $\theta^{1}$ is important, and then there is a decrease of the number of the infected people. Similarly, in Figure 4 , as the control $\theta^{1}$ is important, the number of removed people increases. In fact, this is due to the fact that $K$ has an impact on the values of the control $\theta^{1}$ from the control characterization (9). Finally, these results reinforce the idea that the most important role of the awareness control $\theta^{1}$ is to increase the number of the removed people $R^{1}$. 

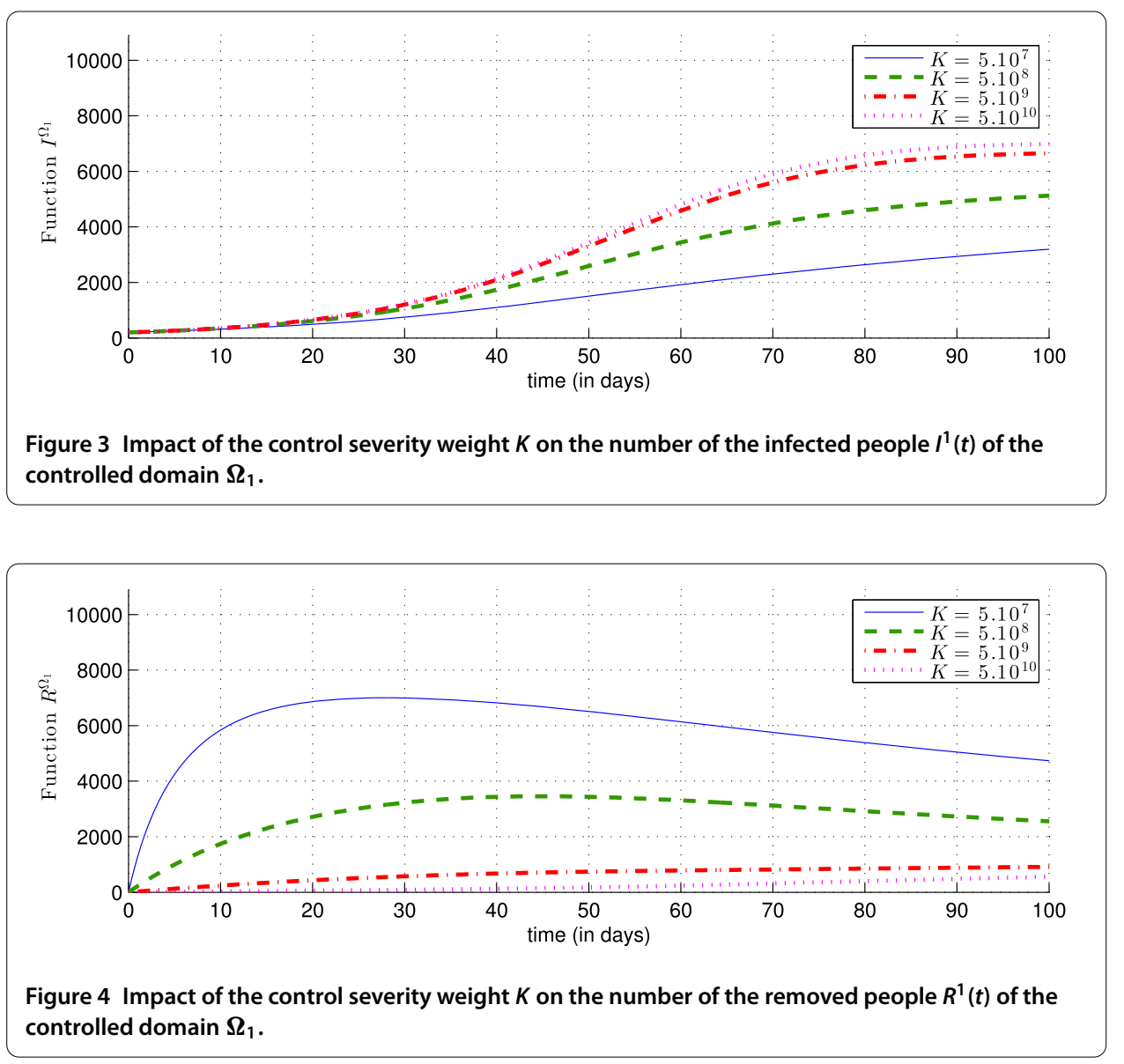

\section{Model with awareness and travel-blocking controls}

\subsection{Presentation of the model}

Let $I=\{1, \ldots, p\}$, and denote by $I_{H} \subset I$ the set of indices of domains at high-risk. This can be a population of homosexuals or prostitutes in a domain or any other population whose behaviors make it high risk to spread HIV. We introduce a control $v^{j \Omega_{k}}$ (or just $v^{j k}$, for simplicity) that characterizes the travel-blocking operation, in order to block all movements from domains $\Omega_{k}, k \in I_{H}$ to the controlled domain $\Omega_{j}$, given by

$$
\begin{cases}v^{j k} \neq 0, & \forall k \in I_{H}, k \neq j \\ v^{j k}=0, & \text { elsewhere. }\end{cases}
$$

Then the multi-domains SIR model will be given as follows:

$$
\begin{aligned}
& \frac{d S^{j}}{d t}=\Gamma_{j}-\sum_{k=1}^{p}\left(1-v^{j k}\right) \beta_{j k} \frac{I^{k}}{I^{k}+S^{k}} S^{j}-\mu_{j} S^{j}-\theta^{j} S^{j}, \\
& \frac{d I^{j}}{d t}=\sum_{k=1}^{p}\left(1-v^{j k}\right) \beta_{j k} \frac{I^{k}}{I^{k}+S^{k}} S^{j}-\gamma_{j} I^{j}-\mu_{j} I^{j}, \\
& \frac{d R^{j}}{d t}=\gamma_{j} I^{j}-\mu_{j} R^{j}+\theta^{j} S^{j} .
\end{aligned}
$$




\subsection{Optimal control problem}

The problem here is to minimize the objective functional given by

$$
J\left(\theta^{j}, v^{j}\right)=\int_{0}^{t_{f}}\left(A_{1} I^{j}(t)-A_{2} R^{j}(t)+\frac{K}{2}\left(\theta^{j}(t)\right)^{2}+\sum_{k \in I_{H}} \frac{K_{k}}{2}\left(v^{j k}(t)\right)^{2}\right),
$$

where $v^{j}=\left(v^{j k}\right)_{k \in I_{H}}$. The first terms represent the crucial goal of the awareness program, that is, reducing the number of the infected people, and increasing the removed ones, in the controlled domain $\Omega_{j}$. The other terms are systemic costs of the awareness program and travel-blocking operation, respectively. The positive constants $A_{1}, A_{2}, K$ and $K_{k}$ for $k \in I_{H}$, balance the values of the terms. Also the reason behind considering a finite time horizon is that such control program is usually restricted to a limited time window.

We seek optimal controls $\theta^{j *}$ and $v^{j *}$ such that

$$
J\left(\theta^{j *}, v^{j *}\right)=\min \left\{J\left(\theta^{j}, v^{j}\right) \mid \theta^{j} \in \Theta, v^{j} \in V\right\},
$$

where

$$
\begin{aligned}
& \Theta=\left\{\theta^{j} \text { measurable, } \theta^{\max } \leq \theta^{j}(t) \leq \theta^{\max }, t \in\left[0, t_{f}\right]\right\}, \\
& V=\left\{v^{j} \text { measurable, } v^{\min _{k}} \leq v^{j k}(t) \leq v^{\max _{k}}, t \in\left[0, t_{f}\right], k \in I_{H}\right\},
\end{aligned}
$$

where $\left.\left(\theta^{\min }, \theta^{\max }, v^{\min _{k}}, v^{\max _{k}}\right) \in\right] 0,1\left[{ }^{4}\right.$, for $k \in I_{H}$. The necessary conditions that an optimal control problem must satisfy come from Pontryagin's maximum principle [50]. This principle converts (14)-(11)-(13) into a problem of minimizing point-wise a Hamiltonian $H$, with respect to $\theta^{j}$ and $v^{j k}$ for $k \in I_{H}$, given by

$$
\begin{aligned}
H= & A_{1} I^{j}-A_{2} R^{j}+\frac{K}{2}\left(\theta^{j}\right)^{2}+\sum_{k \in I_{H}} \frac{K_{k}}{2}\left(v^{j k}\right)^{2} \\
& +\lambda_{1}^{j}\left[\Gamma_{j}-\sum_{k=1}^{p}\left(1-v^{j k}\right) \beta_{j k} \frac{I^{k}}{I^{k}+S^{k}} S^{j}-\mu_{j} S^{j}-\theta^{j} S^{j}\right] \\
& +\lambda_{2}^{j}\left[\sum_{k=1}^{p}\left(1-v^{j k}\right) \beta_{j k} \frac{I^{k}}{I^{k}+S^{k}} S^{j}-\gamma_{j} I^{j}-\mu_{j} I^{j}\right] \\
& +\lambda_{3}^{j}\left[\gamma_{j} I^{j}-\mu_{j} R^{j}+\theta^{j} S^{j}\right] .
\end{aligned}
$$

By applying Pontryagin's maximum principle [50], and assuming the existence result for the optimal control from Theorem 1, we obtain the following.

Theorem 3 There exist optimal controls $\theta^{j *}$ and $\left(v^{j k}\right)_{k \in I_{H}}$ and corresponding solutions $S^{j *}$, $I^{j *}$, and $R^{j *}$, which minimize $J\left(\theta^{j},\left(v^{j k}\right)_{k \in I_{H}}\right)$ over $\Theta$. Furthermore, there exist adjoint functions, $\lambda_{1}^{j}(t), \lambda_{2}^{j}(t)$, and $\lambda_{3}^{j}(t)$, such that

$$
\begin{aligned}
& \dot{\lambda}_{1}^{j}=-\left[\left(\frac{\beta_{j j} S^{j *} I^{j *}}{\left(I^{j *}+S^{j *}\right)^{2}}-\sum_{k=1}^{p}\left(1-v^{j k}\right) \beta_{j k} \frac{I^{k *}}{I^{k *}+S^{k *}}\right)\left(\lambda_{1}^{j}-\lambda_{2}^{j}\right)-\mu_{j} \lambda_{1}^{j}+\theta^{j}\left(\lambda_{3}^{j}-\lambda_{1}^{j}\right)\right], \\
& \dot{\lambda}_{2}^{j}=-\left[A_{1}+\frac{\beta_{j j} S^{j * 2}}{\left(I^{* *}+S^{j *}\right)^{2}}\left(\lambda_{2}^{j}-\lambda_{1}^{j}\right)-\left(\gamma_{j}+\mu_{j}\right) \lambda_{2}^{j}+\gamma_{j} \lambda_{3}^{j}\right],
\end{aligned}
$$




$$
\dot{\lambda}_{3}^{j}=-\left[-A_{2}-\mu_{j} \lambda_{3}^{j}\right]
$$

with transversality conditions

$$
\lambda_{i}^{j}\left(t_{f}\right)=0, \quad i=1,2,3
$$

The following characterization holds:

$$
\theta^{j *}(t)=\max \left\{\min \left\{\left(\lambda_{1}^{j}(t)-\lambda_{3}^{j}(t)\right) \frac{S^{j *}(t)}{K}, \theta^{\max }\right\}, \theta^{\min }\right\},
$$

and for $l \in I_{H}$

$$
v^{j l *}(t)=\max \left\{\min \left\{\frac{\beta_{j l} I^{l *} S^{j *}\left(\lambda_{2}^{j}(t)-\lambda_{1}^{j}(t)\right)}{K_{l}\left(I^{l *}+S^{l *}\right)}, v^{\max _{l}}\right\}, v^{\min _{l}}\right\} .
$$

Proof By using the same proof of Theorem 1, we show the existence of optimal controls. Applying Pontryagin's maximum principle and (10) we obtain

$$
\begin{aligned}
\dot{\lambda}_{1}^{j}= & -\frac{d H}{d S^{j}} \\
= & -\left[\left(\frac{\beta_{j j} S^{j *} I^{j *}}{\left(I^{j *}+S^{j *}\right)^{2}}-\sum_{k=1}^{p}\left(1-v^{j k}\right) \beta_{j k} \frac{I^{k *}}{I^{k *}+S^{k *}}\right)\right. \\
& \left.\times\left(\lambda_{1}^{j}-\lambda_{2}^{j}\right)-\mu_{j} \lambda_{1}^{j}+\theta^{j}\left(\lambda_{3}^{j}-\lambda_{1}^{j}\right)\right], \quad \lambda_{1}^{j}\left(t_{f}\right)=0, \\
\dot{\lambda}_{2}^{j}= & -\frac{d H}{d I^{j}}=-\left[A_{1}+\frac{\beta_{j j} j^{j * 2}}{\left(I^{j *}+S^{j *}\right)^{2}}\left(\lambda_{2}^{j}-\lambda_{1}^{j}\right)-\left(\gamma_{j}+\mu_{j}\right) \lambda_{2}^{j}+\gamma_{j} \lambda_{3}^{j}\right], \quad \lambda_{2}^{j}\left(t_{f}\right)=0, \\
\dot{\lambda}_{3}^{j}= & -\frac{d H}{d R^{j}}=-\left[-A_{2}-\mu_{j} \lambda_{3}^{j}\right], \quad \lambda_{3}^{j}\left(t_{f}\right)=0,
\end{aligned}
$$

evaluated at the optimal controls $\theta^{j *}$ and $v^{j k *}$, for $k \in I_{H}$, and corresponding states $S^{j *}, I^{j *}$, and $R^{j *}$, which results in the stated adjoint system (17)-(19) and (20), [53]. By considering the optimality conditions,

$$
\frac{d H}{d \theta^{j}}=0 \quad \text { and } \quad \frac{d H}{d v^{j k}}=0, \quad \text { for } k \in I_{H},
$$

and solving for $\theta^{j}$, for example, we find

$$
\frac{d H}{d \theta^{j}}=K \theta^{j}-\lambda_{1}^{j} S^{j}+\lambda_{3}^{j} S^{j}=0 .
$$

Taking into account the bounds on the controls in (15) and (16), we deduce that

$$
\theta^{j *}(t)=\max \left\{\min \left\{\left(\lambda_{1}^{j}(t)-\lambda_{3}^{j}(t)\right) \frac{S^{j *}(t)}{K}, \theta^{\max }\right\}, \theta^{\min }\right\}
$$

and for $l \in I_{H}$

$$
v^{j *}(t)=\max \left\{\min \left\{\frac{\beta_{j l} I^{l *} S^{j *}\left(\lambda_{2}^{j}(t)-\lambda_{1}^{j}(t)\right)}{K_{l}\left(I^{l *}+S^{l *}\right)}, v^{\max _{l}}\right\}, v^{\min _{l}}\right\} .
$$


Table 2 Parameters values of $\Gamma, \beta, \mu$, and $\gamma$ utilized for the resolution of the SIR multi-domains differential system (11)-(13), and then leading to simulations obtained from Figure 5 to Figure 7 , with the initial conditions $S_{0}, I_{0}$, and $R_{0}$ associated to the three regions $\Omega_{1}, \Omega_{2}$, and $\Omega_{3}$

\begin{tabular}{rrrlrlll}
\hline & $\boldsymbol{S ( 0 )}$ & $\boldsymbol{I ( 0 )}$ & $\boldsymbol{R ( 0 )}$ & $\boldsymbol{\Gamma}$ & $\boldsymbol{\beta}$ & $\boldsymbol{\mu}$ & $\boldsymbol{\gamma}$ \\
\hline$\Omega_{1}$ & 10,000 & 200 & 0 & 100 & 0.04 & 0.012 & 0.002 \\
$\boldsymbol{\Omega}_{2}$ & 9,000 & 100 & 0 & 180 & 0.05 & 0.013 & 0.001 \\
$\boldsymbol{\Omega}_{3}$ & 9,500 & 50 & 0 & 80 & 0.001 & 0.0144 & 0.002 \\
\hline
\end{tabular}

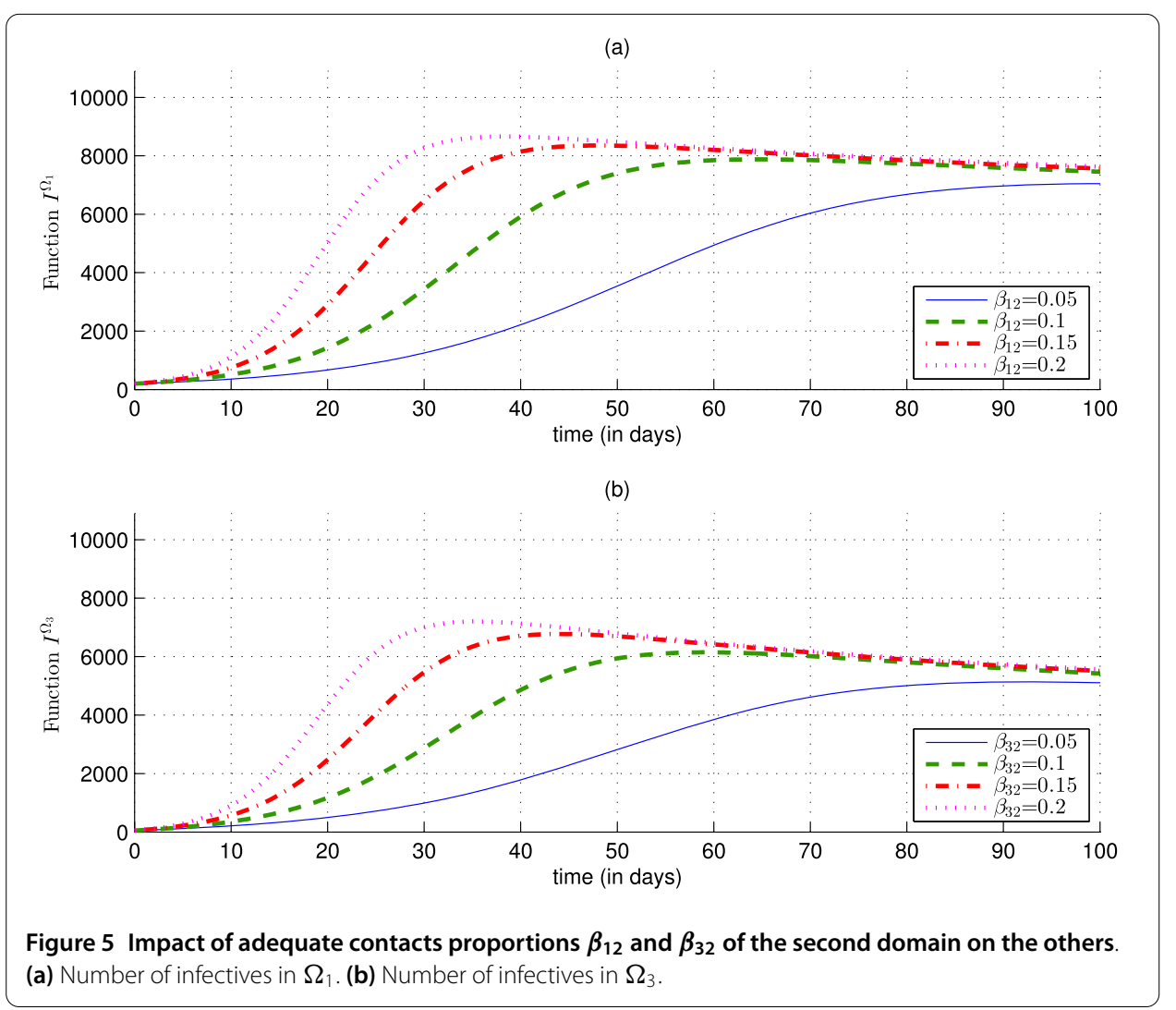

\subsection{Numerical simulation}

Here, a third domain is added, as shown in Table 2. $I=\{1,2,3\}$, and we are interested in controlling $\Omega_{1}$ by assuming that $\Omega_{2}$ is at a high risk of infection $I_{H}=\{2\}$. We introduce a second control $v^{12}$ given by (22), in order to reduce the entry of infected people from domain $\Omega_{2}$. The same data associated to $\Omega_{1}$ and $\Omega_{2}$ in Table 1 are used here to compare between the case when there was no travel-blocking yet and when $v^{12}$ is added.

We should note that in the case of HIV/AIDS, the spatial dynamics of infection occur in close regions. Thus, the epidemic often spreads in very close domains such as neighborhoods, neighboring towns or neighboring cities. By assuming that $\Omega_{1}, \Omega_{2}$, and $\Omega_{3}$ are very close domains, $\beta_{11}=\beta_{21}=\beta_{31}$, which means that $\Omega_{1}$ is accessible from $\Omega_{2}$ and $\Omega_{3}$ with the same proportion, and similarly for the other two domains.

Figure 5 is added here to show the importance of another control associated to travelblocking operations, instead of following only an awareness strategy. In fact, the number of infected people in $\Omega_{1}$ and $\Omega_{2}$ is increased by hosting people coming from $\Omega_{2}$, and we can see the impact of adequate contacts proportions $\beta_{12}$ and $\beta_{32}$ on the behavior of states 


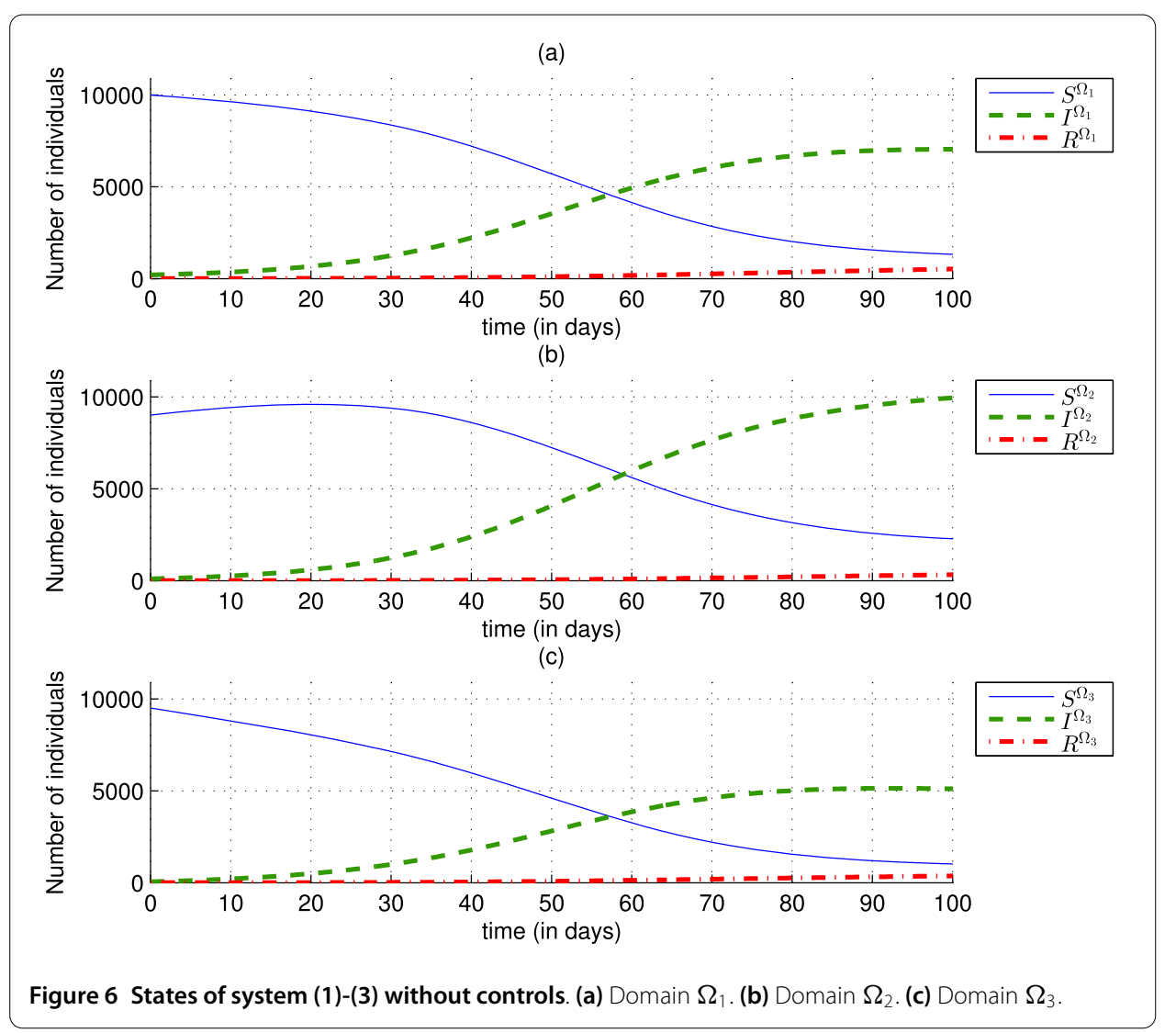

of (17)-(13) in $\Omega_{1}$ and $\Omega_{3}$, and we deduce that the higher $\beta_{12}$ or $\beta_{32}$, the higher the number of infected people in the other domains, becoming huge.

Because of the impact of the adequate contacts proportion associated to only one domain on neighbor domains, by increasing the number of its infected people via travel, as shown in Figure 5, and in order to show the advantage of the travel-blocking control, $v^{12}$, in decreasing the number of infected people who travel to the first domain from the second, we deduce from Figure 7 that the number of infected people in the first domain decreases from 6,190 when there was no control yet in Figure 6, and from 3,800 when the control $\theta^{1}$ is introduced alone in Figure 2, toward a smaller number close to 2,000 when the control $v^{12}$ is added, and which proves that the travel-blocking operation was successful to prevent the disease from spreading. We should note that the higher $v^{12}$, the more we obtain good results regarding the evolution of the proportion of adequate contacts. In fact, it is easy to deduce from the control system (11)-(13) that, when $v^{12}$ is closer to 1 , the term ' $\left(1-v^{12}\right) \beta_{12} \frac{I^{2}}{I^{2}+S^{2}} S^{1}$ ' converges to 0 and when $v^{12}$ is far from 1 , the term ' $\left(1-v^{12}\right) \beta_{12} \frac{I^{2}}{I^{2}+S^{2}} S^{1}$, participates in an increase of the $I^{1}$ function. In contrast, the higher $\theta^{1}$ and far from 0 , the more we can obtain satisfactory results regarding the evolution of the number of the removed people.

Figure 8 presents the simulation of $\theta^{1}$ and $v^{12}$, and we can see that $\theta^{1}$ varies from 0.19 to 0.08 and $v^{12}$ varies from 0.9 to 0.05 , which proves that with this small $v^{12}$ close to 0 , the decrease of the number of infected people in Figure 7 is more important than the case when there was no travel-blocking yet. This proves the effectiveness of our approach in a political sense, where with a negligible blocking of the population of the second domain, 


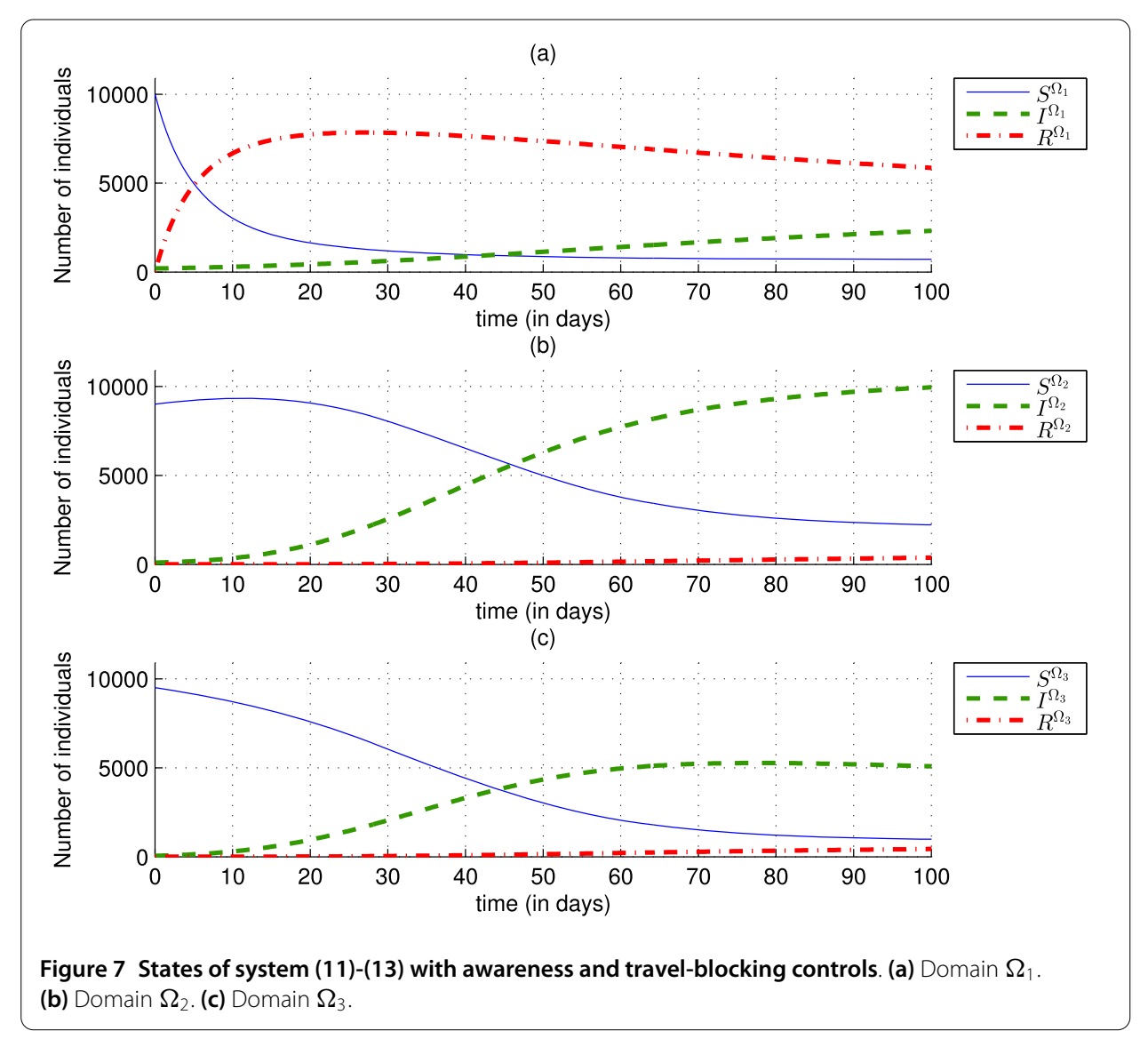

the global goal is reached. As shown in Figure 8, the travel-blocker control can be noticed only about 10 days before it takes a small value close to zero.

In addition, the effect of the lacking amount of the function $\theta^{1}$ in Figure 2 compared to the simulation in Figure 7, could be replaced by the effect of the control $v^{12}$, and by introducing both controls $\theta^{1}$ and $v^{12}$, we would obtain better results, which prove the importance of the travel-blocking control. In general, there does not occur a very big change of the control function $\theta^{1}$, from the case when there is no control term $v^{12}$ yet, to the case when $v^{12}$ is introduced into (11)-(13) as we can see in Figure 8.

Figure 9 shows the impact of the proportion of adequate contacts $\beta_{12}$ on the shapes of the controls $\theta^{1}$ and $v^{12}$. In fact, we can see from this figure that as $\beta_{12}$ is small, $v^{12}$ is small and $\theta^{1}$ is important. This could be explained by the fact that the awareness strategy is more effective when there is less contact between $S^{1}$ and $I^{2}$, and then also the travel-blocking strategy becomes less important to follow.

\section{Conclusion}

A recent study has explained that education and awareness programs based on social media could play a major role in HIV/AIDS prevention [55]. In another study [56], the authors have explained that more efforts and educational plans are essentially needed to fight against the HIV/AIDS epidemic, and those plans have to be conducted not only by parents, but also by other parties of the civil society such as pediatric nurses, professionals, and parent associations. Here, a mathematical model for studying the impact of awareness programs on HIV/AIDS outbreak was therefore proposed in a multi-domains SIR model 

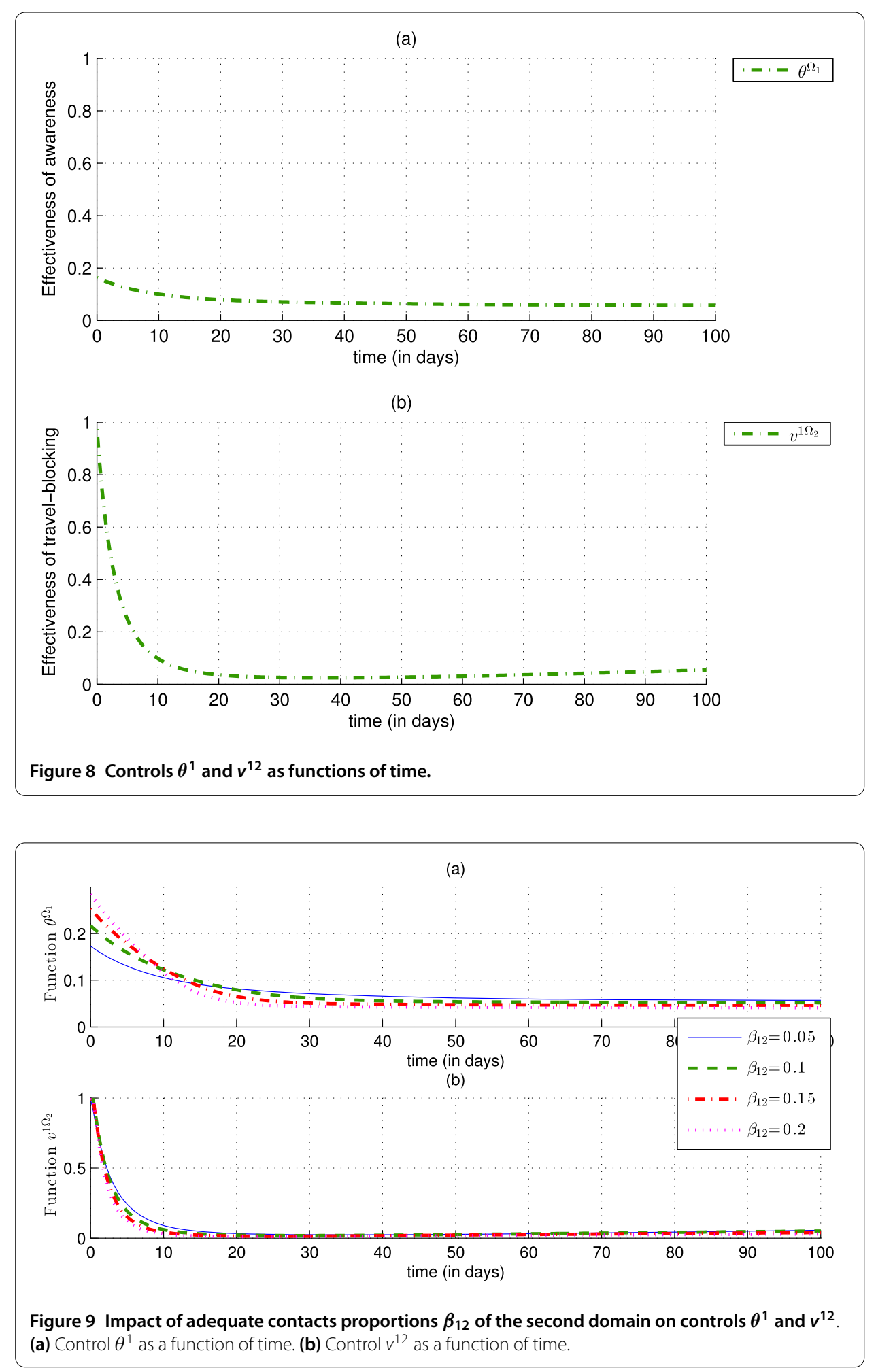

form. A control function was introduced to this multi-domains SIR model to represent the effectiveness of awareness programs. We have identified optimal control strategies for several values of the control severity weights to show the importance and the effectiveness of our approach in controlling the infection spread. Numerical results, associated to this first 
part, showed the effectiveness of this awareness strategy while the targeted region, aiming to control, was still sensitive to the adequate contacts proportion of domains at high risk. The awareness programs alone are not always sufficient to change sexual practices, as concluded by Hoehn et al. in [57], but a comprehensive strategy is needed to decrease the transmission of the epidemic. Therefore, we extended the first part to a second part by adding a travel-blocking control, wherein we controlled contacts between susceptibles of the controlled domain (by awareness) and infectives of domains at high risk of infection with HIV/AIDS. Optimal control strategies were identified. We showed the advantage of each case studied with a comparison between the numerical simulations they provided. Control programs that follow these strategies can effectively reduce the number of infected cases and increase the number of removed individuals in a desired domain.

\section{Competing interests}

The authors declare that they have no competing interests.

Authors' contributions

All authors contributed equally to the writing of this paper. All authors read and approved the final manuscript.

\section{Acknowledgements}

The authors would like to thank all the members of the Editorial Board who were responsible for dealing with this paper, and the anonymous referees for their valuable comments and suggestions, improving the content of this paper. This work is supported by the Systems Theory Network (Réseau Théorie des Systèmes) and Hassan II Academy of Sciences and Technologies-Morocco.

Received: 23 January 2016 Accepted: 14 June 2016 Published online: 29 June 2016

\section{References}

1. AVERTing HIV and AIDS: Global HIV and AIDS statistics. http://www.avert.org/professionals/hiv-around-world/global-statistics (2015)

2. AIDS.GOV: The global HIV/AIDS epidemic. https://www.aids.gov/hiv-aids-basics/hiv-aids-101/global-statistics/ (2015)

3. Laban, A: HIV/AIDS and its impact on education in sub-Saharan Africa: policy initiatives and challenges. The Impact of HIV/AIDS on Education Worldwide 18, 1-26 (2012)

4. Africa Renewal. Women: the face of AIDS in Africa. United Nations Department of Public Information, 18 (2004)

5. Ongaga, K, Ombonga, M: HIV/AIDS education programs in Kenya: contexts of implementation in secondary schools in Kisii county. The Impact of HIV/AIDS on Education Worldwide 18, 27-56 (2012)

6. Aggleton, P, Yankah, E, Crewe, M: Education and HIV/AIDS-30 years on. AIDS Educ. Prev. 23(6), 495-507 (2011)

7. Crewe, M: A pep-talk too far. Reflections on the Power of AIDS Education, University of Pretoria (2004)

8. Joint United Nations Programme on HIV/AIDS (UNAIDS), et al.: Global coalition on women and AIDS. Global campaign for education global AIDS alliance, Geneva, Switzerland. http://www.unaids.org (2002)

9. Akala, FA, Semini, I: Characterizing the HIV/AIDS Epidemic in the Middle East and North Africa: Time for Strategic Action. World Bank, Washington (2010)

10. Bundy, D, Patrikios, A, Mannathoko, C, Tembon, A, Manda, S, Sarr, B, Drake, L: Accelerating the Education Sector Response to HIV: Five Years of Experience from Sub-Saharan Africa. The World Bank, Washington (2010)

11. Catalán, J, Hedge, B, Sherr, L: The Impact of AIDS: Psychological and Social Aspects of HIV Infection. Harwood Academic, Reading (1997)

12. Zakary, O, Rachik, M, Elmouki, I: On the analysis of a multi-regions discrete SIR epidemic model: an optimal control approach. Int. J. Dyn. Control (2016). doi:10.1007/s40435-016-0233-2

13. Arino, J, Van Den Driessche, P: The basic reproduction number in a multi-city compartmental epidemic model. In: Positive Systems, pp. 135-142. Springer, Berlin (2003)

14. Arino, J, Van den Driessche, P: A multi-city epidemic model. Math. Popul. Stud. 10(3), 175-193 (2003)

15. Arino, J, Jordan, R, Van den Driessche, P: Quarantine in a multi-species epidemic model with spatial dynamics. Math. Biosci. 206(1), 46-60 (2007)

16. Allen, LJS, Jones, MA, Martin, CF: A discrete-time model with vaccination for a measles epidemic. Math. Biosci. 105(1), 111-131 (1991)

17. Baize, S, Pannetier, D, Oestereich, L, Rieger, T, Koivogui, L, Magassouba, N, Soropogui, B, Saliou Sow, M, Keïta, S, De Clerck, H et al.: Emergence of Zaire Ebola virus disease in Guinea. N. Engl. J. Med. 371(15), 1418-1425 (2014)

18. World Health Organ. Who, Liberian health authorities confirm two cases of Ebola. Reuters. Retrieved march 30, (2014)

19. Jung, E, Lenhart, S, Feng, Z: Optimal control of treatments in a two-strain tuberculosis model. Discrete Contin. Dyn. Syst., Ser. B 2(4), 473-482 (2002)

20. Moualeu, DP, Weiser, M, Ehrig, R, Deuflhard, P: Optimal control for a tuberculosis model with undetected cases in Cameroon. Commun. Nonlinear Sci. Numer. Simul. 20(3), 986-1003 (2015)

21. Silva, CJ, Torres, DFM: Optimal control for a tuberculosis model with reinfection and post-exposure interventions. Math. Biosci. 244(2), 154-164 (2013) 
22. Whang, S, Choi, S, Jung, E: A dynamic model for tuberculosis transmission and optimal treatment strategies in South Korea. J. Theor. Biol. 279(1), 120-131 (2011)

23. Agusto, FB, Adekunle, Al: Optimal control of a two-strain tuberculosis-HIV/AIDS co-infection model. Biosystems 119, 20-44 (2014)

24. Nim Kim, B, Nah, K, Chu, C, Uk Ryu, S, Han Kang, Y, Kim, Y: Optimal control strategy of Plasmodium vivax malaria transmission in Korea. Osong Public Health Res. Perspect. 3(3), 128-136 (2012)

25. Prosper, O, Ruktanonchai, N, Martcheva, M: Optimal vaccination and bednet maintenance for the control of malaria in a region with naturally acquired immunity. J. Theor. Biol. 353, 142-156 (2014)

26. Zakary, O, Rachik, M, Elmouki, I: On the impact of awareness programs in HIV/AIDS prevention: an SIR model with optimal control. Int. J. Comput. Appl. 133(9), 1-6 (2016)

27. Renee Fister, K, Lenhart, S, McNally, JS: Optimizing chemotherapy in an HIV model. Electron. J. Differ. Equ. 1998(32), 1 (1998)

28. Yang, Y, Xiao, Y, Wu, J: Pulse HIV vaccination: feasibility for virus eradication and optimal vaccination schedule. Bull. Math. Biol. 75(5), 725-751 (2013)

29. Kwon, H-D, Lee, J, Yang, S-D: Optimal control of an age-structured model of HIV infection. Appl. Math. Comput. 219(5), 2766-2779 (2012)

30. Roshanfekr, M, Hadi Farahi, M, Rahbarian, R: A different approach of optimal control on an HIV immunology model. Ain Shams Eng. J. 5(1), 213-219 (2014)

31. Zhou, Y, Liang, Y, Wu, J: An optimal strategy for HIV multitherapy. J. Comput. Appl. Math. 263, 326-337 (2014)

32. Adams, BM, Banks, HT, Davidian, M, Kwon, H-D, Tran, HT, Wynne, SN, Rosenberg, ES: HIV dynamics: modeling, data analysis, and optimal treatment protocols. J. Comput. Appl. Math. 184(1), 10-49 (2005)

33. Costanza, V, Rivadeneira, PS, Biafore, FL, D'Attellis, CE: Optimizing thymic recovery in HIV patients through multidrug therapies. Biomed. Signal Process. Control 8(1), 90-97 (2013)

34. Zakary, O, Rachik, M, Elmouki, I: On effectiveness of an optimal antiviral bitherapy in HBV-HDV coinfection model. Int. J. Comput. Appl. 127(12), 1-10 (2015)

35. Chakrabarty, SP, Raj Joshi, H: Optimally controlled treatment strategy using interferon and ribavirin for hepatitis C. J. Biol. Syst. 17(1), 97-110 (2009)

36. Græsbøll, K, Enøe, C, Bødker, R, Christiansen, LE: Optimal vaccination strategies against vector-borne diseases. Spat. Spat.-Temporal Epidemiol. 11, 153-162 (2014)

37. Burden, TN, Ernstberger, J, Fister, KR: Optimal control applied to immunotherapy. Discrete Contin. Dyn. Syst., Ser. B $4(1), 135-146(2004)$

38. Ledzewicz, U, Schättler, H: Antiangiogenic therapy in cancer treatment as an optimal control problem. SIAM J. Control Optim. 46(3), 1052-1079 (2007)

39. Castiglione, F, Piccoli, B: Cancer immunotherapy, mathematical modeling and optimal control. J. Theor. Biol. 247(4), 723-732 (2007)

40. Elmouki, I, Saadi, S: Quadratic and linear controls developing an optimal treatment for the use of BCG immunotherapy in superficial bladder cancer. Optim. Control Appl. Methods 37(1), 176-189 (2016)

41. Martin, RB: Optimal control drug scheduling of cancer chemotherapy. Automatica 28(6), 1113-1123 (1992)

42. Engelhart, M, Lebiedz, D, Sager, S: Optimal control for selected cancer chemotherapy ODE models: a view on the potential of optimal schedules and choice of objective function. Math. Biosci. 229(1), 123-134 (2011)

43. Yan, X, Zou, Y: Optimal and sub-optimal quarantine and isolation control in SARS epidemics. Math. Comput. Model. 47(1), 235-245 (2008)

44. Agusto, FB: Optimal isolation control strategies and cost-effectiveness analysis of a two-strain avian influenza model. Biosystems 113(3), 155-164 (2013)

45. Brown, VL, White, KJ: The role of optimal control in assessing the most cost-effective implementation of a vaccination programme: HPV as a case study. Math. Biosci. 231(2), 126-134 (2011)

46. Su, Y, Sun, D: Optimal control of anti-HBV treatment based on combination of traditional Chinese medicine and western medicine. Biomed. Signal Process. Control 15, 41-48 (2015)

47. Buonomo, B, Lacitignola, D, Vargas-De-León, C: Qualitative analysis and optimal control of an epidemic model with vaccination and treatment. Math. Comput. Simul. 100, 88-102 (2014)

48. Lowden, J, Neilan, RM, Yahdi, M: Optimal control of vancomycin-resistant enterococci using preventive care and treatment of infections. Math. Biosci. 249, 8-17 (2014)

49. Apreutesei, N, Dimitriu, G, Strugariu, R: An optimal control problem for a two-prey and one-predator model with diffusion. Comput. Math. Appl. 67(12), 2127-2143 (2014)

50. Pontryagin, LS: Mathematical Theory of Optimal Processes. CRC Press, New York (1987)

51. Fleming, WH, Rishel, RW: Deterministic and Stochastic Optimal Control. Springer, New York (1975)

52. Lukes, DL: Differential Equations: Classical to Controlled, vol. 162. Academic Press, New York (1982)

53. Kamien, Ml, Schwartz, NL: Dynamic Optimisation. The Calculus of Variations and Optimal Control in Economics and Management, 2nd edn. North Holland, New York (1991)

54. Lenhart, S, Workman, JT: Optimal Control Applied to Biological Models. CRC Press, New York (2007)

55. Chandran, D: Social media and HIV/AIDS: implications for social work education. J. Soc. Work Educ. 35(3), 333-343 (2016)

56. Mahat, G, Scoloveno, MA, Scoloveno, R: HIV/AIDS knowledge, self-efficacy for limiting sexual risk behavior and parental monitoring. J. Pediatr. Nurs. 31(1), e63-e69 (2016)

57. Hoehn, EF, FitzGerald, MR, Bhatt, SR, Robinson, VM, Lippe, JE, Reed, JL: Do adolescents with higher knowledge of HIV have lower sexual risk behaviors? Pediatr. Emerg. Care (2016) 